\title{
Defective positioning in granulomas but not lung-homing limits CD4 T-cell interactions with Mycobacterium tuberculosis-infected macrophages in rhesus macaques
}

\author{
KD Kauffman $^{1}$, MA Sallin ${ }^{1}$, S Sakai $^{1}$, O Kamenyeva $^{2}$, J Kabat $^{2}$, D Weiner $^{3}$, M Sutphin $^{3}$, D Schimel $^{3}$, L Via $^{3}$, \\ CE Barry III ${ }^{3}$, T Wilder-Kofie ${ }^{4}$, I Moore ${ }^{4}, \mathrm{R}_{\text {Moore }}{ }^{4}$ and DL Barber ${ }^{1}$
}

Protection against Mycobacterium tuberculosis (Mtb) infection requires CD4 Tcells to migrate into the lung and interact with infected macrophages. In mice, less-differentiated $\mathrm{CXCR} 3^{+} \mathrm{CD} 4$ Tcells migrate into the lung and suppress growth of Mtb, whereas CX3CR1 ${ }^{+}$terminally differentiated Th1 cells accumulate in the blood vasculature and do not control pulmonary infection. Here we examine CD4 T-cell differentiation and lung homing during primary Mtb infection of rhesus macaques. Mtb-specific CD4 T cells simultaneously appeared in the airways and blood $\sim 21-28$ days post exposure, indicating that recently primed effectors are quickly recruited into the lungs after entering circulation. Mtb-specific CD4 T cells in granulomas display a tissue-parenchymal CXCR3 ${ }^{+}$CX3CR1 ${ }^{-}$PD $-1{ }^{\text {hi } C T L A-4 ~}{ }^{+}$phenotype. However, most granuloma CD4 Tcells are found within the outer lymphocyte cuff and few localize to the myeloid cell core containing the bacilli. Using the intravascular stain approach, we find essentially all Mtb-specific CD4 T cells in granulomas have extravasated across the vascular endothelium into the parenchyma. Therefore, it is unlikely to be that lung-homing defects introduced by terminal differentiation limit the migration of CD4 T cells into granulomas following primary Mtb infection of macaques. However, intralesional positioning defects within the granuloma may pose a major barrier to T-cell-mediated immunity during tuberculosis.

\section{INTRODUCTION}

CD4 T cells are critical for control of $M$. tuberculosis infection. ${ }^{1-4}$ In order to mediate protection, $\mathrm{CD} 4 \mathrm{~T}$ cells must recognize major histocompatibility complex class II on the surface of infected macrophages within the lung tissue and deliver signals that instruct macrophages to restrict growth of their ingested bacilli through direct cell-to-cell interactions. ${ }^{5}$ Therefore, the ability of Mycobacterium tuberculosis (Mtb)-specific CD4 T cells to migrate into the site of infection and interact with infected antigen presenting cells is key to protection during $\mathrm{Mtb}$ infection. In mice, interleukin-12 and T-bet-dependent CD4 $\mathrm{T}$-cell differentiation generates distinct subsets of Mtb-specific
Th1 cells with varying levels of protective capacity after primary infection. CXCR $^{+}$Th1 cells that express intermediate levels of T-bet are able to efficiently migrate out of the blood vasculature into the lung, expand and can adoptively transfer protection to infected T-cell-deficient recipient mice. ${ }^{6-9}$ In contrast, $\mathrm{T}$ cells that undergo extensive Th1 differentiation become $\mathrm{CX} 3 \mathrm{CR} 1^{+} \mathrm{KLRG}^{+}{ }^{+}$T-bet ${ }^{\text {high }}$ terminal effector cells that cannot expand, poorly exit the pulmonary vasculature into the tissue parenchyma, and do not adoptively transfer protection. Although T-bet expression in CD4 T cells is required for interferon (IFN)- $\gamma$ production and host protection, ${ }^{9,10}$ T-bet haploinsufficient mice do not generate KLRG1 ${ }^{+}$

\footnotetext{
${ }^{1}$ T Lymphocyte Biology Unit, Laboratory of Parasitic Diseases, National Institute of Allergy and Infectious Diseases, National Institutes of Health, Bethesda, Maryland, USA. ${ }^{2}$ Biological Imaging Section, Research Technologies Branch, National Institute of Allergy and Infectious Diseases, National Institutes of Health, Bethesda, Maryland, USA. ${ }^{3}$ Tuberculosis Research Section, Laboratory of Clinical Infectious Diseases, National Institute of Allergy and Infectious Disease, National Institutes of Health, Bethesda, Maryland, USA and ${ }^{4}$ Comparative Medicine Branch, National Institute of Allergy and Infectious Diseases, National Institutes of Health, Bethesda, Maryland, USA. Correspondence: DL Barber (barberd@niaid.nih.gov)
} 
CD4 T cells and are more resistant to Mtb infection compared to wild-type mice. ${ }^{11}$

Based on these observations in the murine model of Mtb infection, there is a hypothesis that the differentiation state of $\mathrm{CD} 4 \mathrm{~T}$ cells is a major determinant of their protective capacity against Mtb infection and vaccination should aim to selectively promote the generation of less-differentiated CD4 T cells. ${ }^{12}$ Indeed, it has been found in mice that vaccine strategies that generate memory $\mathrm{T}$-cell populations that can resist terminal effector cell formation upon Mtb challenge are more protective. ${ }^{13-15}$ It is not known, however, if the generation of Ag-specific non-protective terminal effector cells occurs in other species following $\mathrm{Mtb}$ infection or to what extent defects in $\mathrm{CD} 4 \mathrm{~T}$-cell migration into the lung due to terminal differentiation limits the overall protective quality of the Mtb-specific effector cell population.

After CD4 T cells extravasate across lung blood vascular endothelium, there is a subsequent phase of migration within the tissue as CD4 T cells locate Mtb-infected macrophages. In mice, there is relatively little structure to the organization of immune cells that cluster around sites of bacterial replication and true human-like granulomas do not form, at least not in the most commonly used inbred mouse strains. Therefore, the mouse model is not optimal for the study of intralesional leukocyte positioning and trafficking, and little is known about this aspect of T-cell function during tuberculosis in higher mammals. In contrast to mice, Mtb-infected non-human primates form complex, human-like granulomas with reproducibly identifiable and clearly demarcated cellular strata with distinct inflammatory microenvironments. ${ }^{16,17}$ Although tuberculosis granulomas can be classified into many subtypes with very different outcomes, in general, $\mathrm{CD}^{+}$cells are abundant in a cuff circumscribing a central macrophage-rich region where the bacteria reside and very few lymphocytes are immediately proximal to infected macrophages. ${ }^{18-20}$ Therefore, both transendothelial diapedesis as well as the intalesional positioning of effector $\mathrm{CD} 4 \mathrm{~T}$ cells are potential points of failure in T-cell trafficking in the setting of tuberculosis.

Here we examine the differentiation state of Mtb-specific effector CD4 T cells generated after low-dose Mtb infection of rhesus macaques. We find that rhesus macaque $\mathrm{CD} 4 \mathrm{~T}$ cells do not undergo terminal differentiation during the clonal expansion phase and display markers of lung tissue-parenchymal cells following pulmonary Mtb infection. Using the intravascular staining technique, we show that $\mathrm{CX} 3 \mathrm{CR} 1{ }^{+} \mathrm{CD} 4$ $\mathrm{T}$ cells found in uninfected rhesus macaques are localized to the

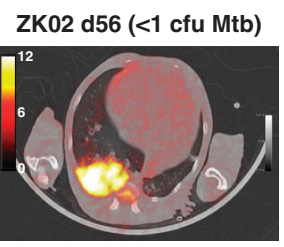

C
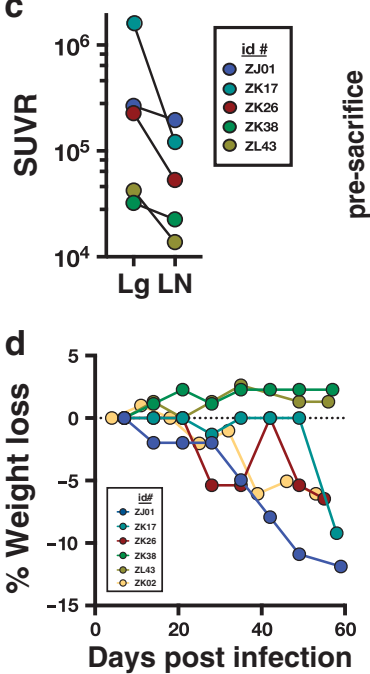

b
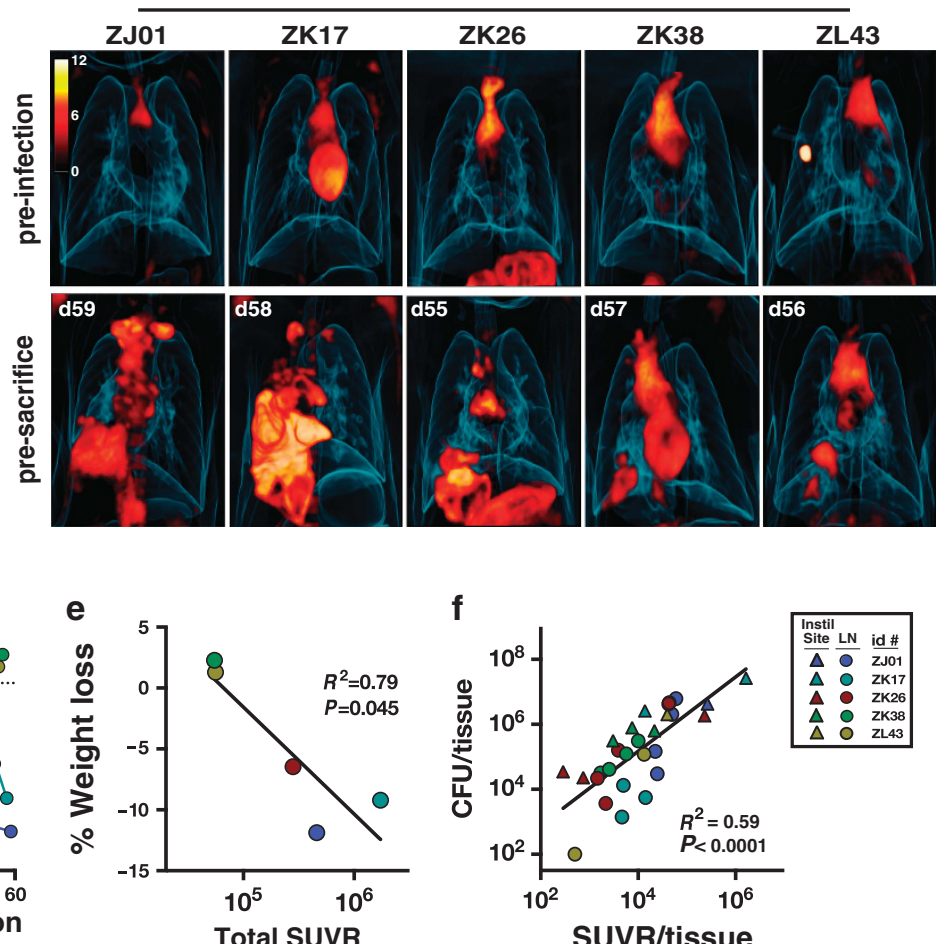

f

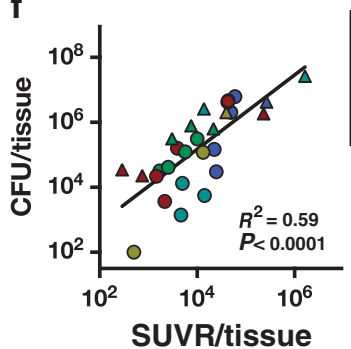

Instil
Site $L N$ id \#

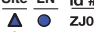

$\begin{array}{lll}\Delta & \circ & \text { ZJ01 } \\ \Delta & \circ & \text { ZK17 }\end{array}$

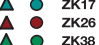

$\stackrel{\Delta}{\Delta}: \mathrm{Z}^{\mathrm{ZK} 38}$

Figure 1 Variable disease severity following ultra-low dose Mtb infection of rhesus macaques. Six rhesus macaques were exposed to >1 cfu MtbErdman strain via bronchoscope. (a) Only one of the six animals became infected (ZKO2) as confirmed by ${ }^{18} \mathrm{FDG}$ positron emission tomographycomputed tomography (PET/CT) scan. (b) The five remaining animals were confirmed negative as described in the text and then re-exposed to $6-10$ cfu. ${ }^{18}$ FDG PET/CT scans were taken pre-infection and $\sim 2$ months post infection, just before necropsy. The day post infection of the scan is indicated in white letters on each scan. It is worth noting that the catheter is visible as a bright spot in the pre-infection scan of animal ZL43. (c) Standard uptake value ratio (SUVR) values were calculated for lung and pulmonary lymph nodes of infected animals. (d) Weight loss was monitored following infection. (e) Correlation between total SUVR in lung and pulmonary lymph nodes and percent weight loss at time of ${ }^{18}$ FDG PET/CT scan. (f) Correlation between SUVR and bacterial loads in individual instillation site lesions and pulmonary lymph nodes. 
lung-associated blood vasculature, indicating that the poor lung-homing of this subset of $\mathrm{CD} 4 \mathrm{~T}$ cells is similar between mice and rhesus macaques. However, Mtb-specific effector cells rarely express CX3CR1 and almost all CD4 $\mathrm{T}$ cells in granulomas have migrated out of the blood vasculature into the tissue parenchyma. We quantify T-cell distribution into different regions of granulomas in macaques infected with Mtb-RFP and find that $\sim 1-5 \%$ of CD4 T cells in granulomas are localized within the myeloid cell rich core containing the bacilli, similar to what has been thought previously. Therefore, although CD4 T cells efficiently enter the lungs of Mtb-infected rhesus macaques, poor migration within granulomas may represent a major limitation in the effectiveness of CD4 T cell-mediated protection against tuberculosis.

\section{RESULTS}

\section{Variable disease severity following ultra-low dose Mtb infection of rhesus macaques}

Six rhesus macaques were exposed to an ultra-low dose of Mtb-Erdman. In this first round of exposure the bacterial inoculum was diluted to an undetectable number of bacteria as measured by colony forming units (CFUs). Nonetheless, one of the six animals (ZK02) displayed evidence of infection. Animal ZK02 developed a large lesion in the lower right lobe at the site of instillation as shown by the ${ }^{18} \mathrm{FDG}$ positron emission tomography-computed tomography (PET/CT) scan on day 56 post infection (Figure 1a). None of the remaining five animals displayed signs of infection. First, they remained healthy and no purified protein derivative (PPD) skin test reactions were detected. Second, we obtained weekly blood and bronchoalveolar lavage (BAL) samples for 6 weeks and could not detect Mtbspecific CD4 T-cell responses by intracellular cytokine staining (data not shown). Lastly, we obtained ${ }^{18}$ FDG PET/CT scans before the second infection attempt and found no evidence of disease (Figure 1b). Therefore, these animals were considered uninfected and were re-exposed to a second instillation of 6-10 $\mathrm{CFU}$ of Mtb-Erdman. All remaining five animals became infected after this second round of exposure. Approximately 2 months following infection, ${ }^{18} \mathrm{FDG}$ PET/CT scans revealed extensive pulmonary inflammation and large lesions in the lower right lobe at the site of instillation and pulmonary lymph nodes in every animal. Although all animals showed a similar pattern of disease, the total PET hot activity in the lung varied by more than 10 -fold. After normalizing the lung ${ }^{18} \mathrm{FDG}$ uptake to a portion of the cylinder muscle, the total standard uptake value ratio (SUVR) for the entire lung ranged from around $3.2 \times 10^{4}$ SUVR (ZK38) to over $1.6 \times 10^{6}$ SUVR (ZK17) (Figure 1c). The total ${ }^{18} \mathrm{FDG}$ avidity from all pulmonary lymph nodes ranged between $1.37 \times 10^{4}$ SUVR (ZL43) through $1.9 \times 10^{5}$ SUVR (ZJ01) (Figure 1c).
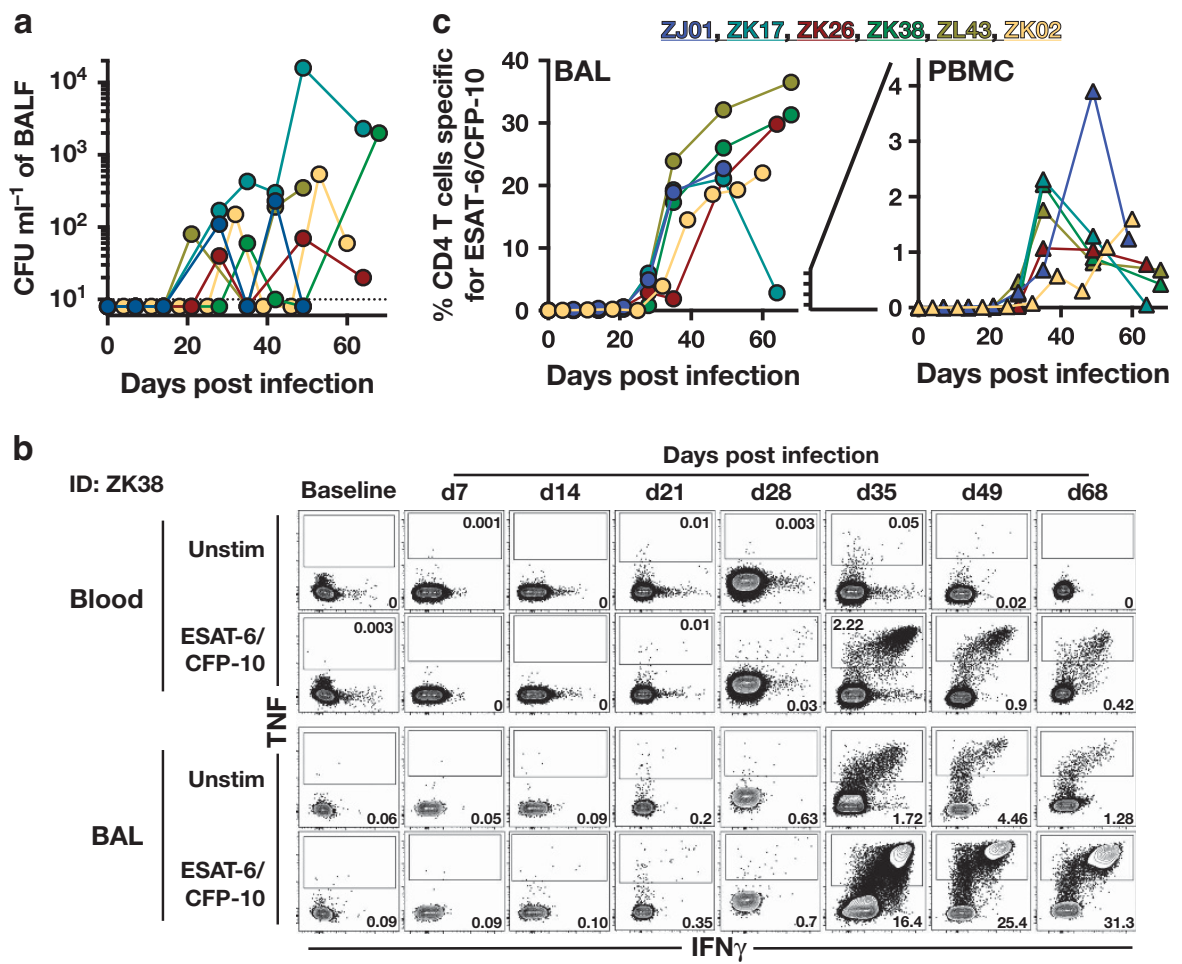

Figure 2 Ag-specific CD4 T cells simultaneously appear in the peripheral blood and airways following Mtb infection. Weekly blood and BALf samples were collected before and following Mtb infection. (a) Bacterial loads were quantified in bronchoalveolar lavage (BAL) fluid. (b) Example fluorescenceactivated cell sorting (FACS) plots showing intracellular cytokine staining for tumor necrosis factor (TNF) and interferon (IFN)- $\gamma$ following a $6 \mathrm{~h}$ in vitro stimulation with ESAT-6/CFP-10 peptide pools. (c) Kinetics of Ag-specific CD4 T cells in blood and BALf following Mtb infection. It is noteworthy that ZK02 was infected with $>1 \mathrm{cfu}$, whereas the other animals were exposed to $6-10 \mathrm{cfu}$ as described in Figure 1. Numbers represent the frequency of TNF ${ }^{+} \mathrm{CD} 4$ T cells following stimulation with ESAT-6/CFP-10 peptide pools. 
The amount of weight loss varied from a slight gain in weight to $>10 \%$ loss by 2 months post infection (Figure 1d). Interestingly, weight loss at the final time point inversely correlated with lung total SUVR (Figure 1e). We also found that PET hot values from individual large lesions at the instillation sites and lymph nodes correlated well with bacterial loads (Figure 1f). This is consistent with previous reports that ${ }^{18}$ FDG uptake in individual granulomas correlates with bacterial burden in Mtb-infected monkeys. ${ }^{21,22}$ Taken together, these data show that an undetectable number of bacteria are able to establish infection and cause disease in some exposed macaques, and after exposure to only 6-10 organisms all animals become infected and develop mild to severe disease by $\sim 2$ months post infection. This highlights the relatively high susceptibility of rhesus macaques to the Erdman strain of Mtb.

\section{Kinetics of Ag-specific CD4 T-cell responses in the airways following Mtb infection}

Blood draws and BAL samples were taken weekly during the course of infection for all six animals. Bacteria first became detectable in the BAL at $\sim$ day 21 post infection and although there was a high degree of variability, all animals had detectable levels of bacteria in the BAL fluid at least at two different time points (Figure 2a). Notably, BAL fluid from animal ZK17, which had the highest ${ }^{18}$ FDG uptake in the lung, was consistently positive for the presence of bacteria after week 4 and showed the highest numbers of bacteria (Figure 2a).

To compare the kinetics of Mtb-specific CD4 T-cell responses in circulation versus the airways, Ag-specific CD4 $\mathrm{T}$ cells were measured by intracellular cytokine staining following a $6 \mathrm{~h}$ stimulation with a mixture of peptide pools from ESAT-6 and CFP-10 (Figure 2b). Antigen-specific CD4 T cells first became detectable in both the blood and BAL at $\sim$ day 21-28 post infection (Figure $\mathbf{2 b}, \mathbf{c}$ ). Strikingly, we observed remarkably high frequencies of $\mathrm{CD} 4 \mathrm{~T}$ cells specific for these two antigens in the BAL, reaching upwards of $\sim 20-35 \%$, as compared with peak frequencies of $\sim 1$ to $4 \%$ in the blood. It is also interesting to note that responses tended to continually increase in the BAL until the time of necropsy, whereas in the blood the peak response was between week 5 to week 7 for most animals. Therefore, the primary Ag-specific effector CD4 T-cell response appears simultaneously in the blood and BAL, and reaches much higher frequencies at the site of infection.

\section{Ag-specific CD4 T-cell responses in tissues of Mtb-infected rhesus macaques}

We next quantified the ESAT-6/CFP-10-specific CD4 T-cell response in different tissues at necropsy (Figure 3a). Similar to the dramatic enrichment for Ag-specific CD4 $\mathrm{T}$ cells we observed in the airways compared with the blood, we found that the frequency of the Mtb-specific CD4 T-cell response was much greater in large instillation site lesions as compared to lymphoid tissues (Figure $3 \mathbf{b}$ ). Individual granulomas were also excised at the time of necropsy and processed for bacterial burden and flow cytometry analysis. As has been previously reported, ${ }^{23}$ there was tremendous variability in the magnitude of the Ag-specific CD4 T-cell response in the granulomas between different animals as well as among granulomas within any individual animal (Figure 3c). Interestingly, animals ZK38 and ZL43, which had no weight loss, the lowest PET hot signals and the largest $\mathrm{CD} 4 \mathrm{~T}$-cell responses in the airways also had the largest CD4 T-cell responses in individual granulomas. In contrast, animals ZJ01 and ZK17, which were clinically the most ill, displayed the most ${ }^{18} \mathrm{FDG}$ uptake, and lost the most weight had very few viable Ag-specific CD4 T cells (Figure 3c). Therefore, in general, animals that maintained robust Mtbspecific CD4 T-cell responses in granulomas fared much better than animals that displayed lower frequencies of specific CD4 $\mathrm{T}$ cells at the time of necropsy.

Bacterial loads in individual granulomas also varied greatly within each animal, with CFU values varying 1-3 logs in individual animals (Figure 3d). Finally, we asked whether the frequency of ESAT-6/CFP-10-specific CD4 T cells correlated with bacterial loads in individual granulomas (Figure 3e). The granulomas with the smallest $\mathrm{CD} 4 \mathrm{~T}$-cell responses tended to be those with either the highest or lowest bacterial load, whereas granulomas with the highest $\mathrm{CD} 4 \mathrm{~T}$-cell responses tended to be those with intermediate CFU values, resulting in a weak Gaussian distribution. Regardless, we did not find evidence that the magnitude of the CD4 T-cell response in individual granulomas is a predictor of bacterial control. However, we cannot rule out the possibility that CD4 T cells specific to other Mtb antigens may be better predictors of bacterial burdens.

\section{Few CX3CR1 expressing Ag-specific CD4 T cells develop during Mtb infection of rhesus macaques}

Ourselves and others have previously demonstrated that the differentiation state of Mtb-specific CD4 T cells has a dramatic impact on their ability to migrate into the lung and control $\mathrm{Mtb}$ growth. ${ }^{7,8} \mathrm{CXCR}^{+}{ }^{+} \mathrm{Mtb}$-specific CD4 $\mathrm{T}$ cells are able to enter the lung tissue and can suppress bacterial growth after adoptive transfer into infected T-cell-deficient recipient mice. In contrast, $\mathrm{CX} 3 \mathrm{CR} 1^{+} \mathrm{CD} 4 \mathrm{~T}$ cells accumulate in the lungassociated blood vasculature, poorly enter the lung tissue, and do not transfer protection to infected T-cell-deficient mice. To test whether the Mtb-specific CD4 $\mathrm{T}$-cell response in rhesus macaques also comprised these two major protective and nonprotective subsets, we measured CXCR3 and CX3CR1 expression on ESAT-6/CFP-10-specific CD4 $\mathrm{T}$ cells in the blood and BAL over the first $\sim 2$ months of infection (Figure 4a,b). We observed that almost all Ag-specific CD4 $\mathrm{T}$ cells expressed CXCR3 in both the blood and BAL, whereas there was very little or no expression of CX3CR1 on Mtbspecific CD4 T cells. The highest level of CX3CR1 expression on Mtb-specific CD4 T cells we observed was in animals ZK26 and ZK17 ( 20-35\%) in the blood at day 21, but the levels dropped off dramatically thereafter. Similarly, the ESAT-6/CFP-10specific CD4 T cells from lymph nodes, large instillation site lesions, and granulomas expressed high levels of CXCR3 but little to no CX3CR1 (Figure 4c,d). Therefore, unlike mice where approximately half of the ESAT-6-specific CD4 T cells express CX3CR1, indicating a terminal effector phenotype, we 
a
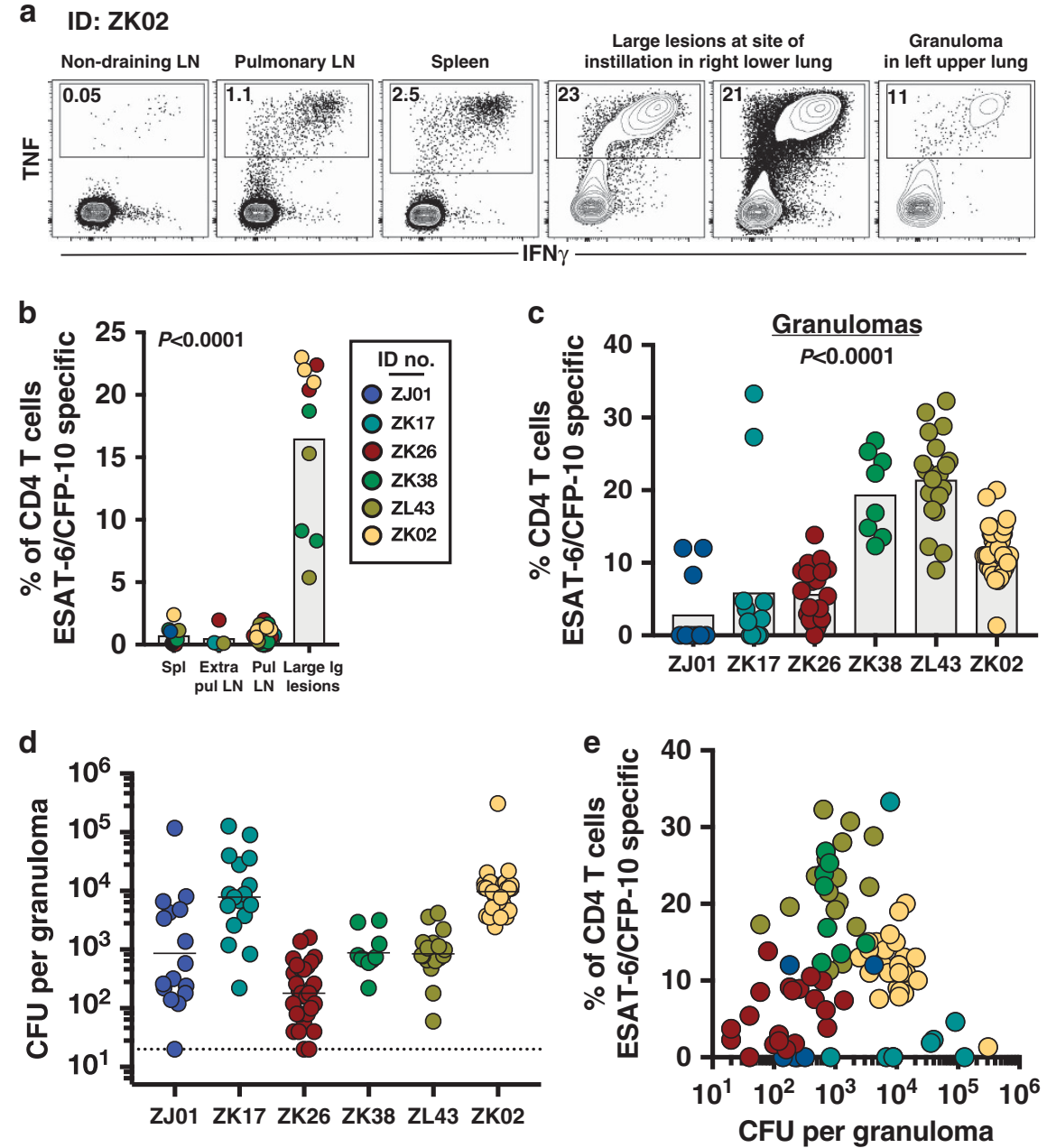

Figure 3 Ag-specific CD4 T cells in lymphoid tissues and pulmonary lesions of Mtb-infected rhesus macaques. Tissues were obtained from animals 2 months post infection. (a,b) Example fluorescence-activated cell sorting (FACS) plots (a) and summary quantification (b) of intracellular cytokine staining of tumor necrosis factor (TNF) and interferon (IFN)- $\gamma$ following a $6 \mathrm{~h}$ in vitro stimulation of cells from instillation site lesions and lymphoid tissue with ESAT6/CFP-10 peptide pools. (c) Ag-specific CD4 T-cell responses in individually resected pulmonary granulomas as quantified by ICC. (d) Bacterial loads in granulomas. (e) Granuloma bacterial burden did not correlate with the percentage of ESAT-6/CFP-10-specific CD4 T cells. $P$-values are from a one-way analysis of variance test.

observed very few CX3CR1 ${ }^{+}$Ag-specific CD4 T cells in Mtbinfected rhesus macaques.

We also measured the expression of CCR5 (another Th1associated chemokine receptor), CCR6 (which has been found on human Mtb-specific CD4 $\mathrm{T}$ cells in latently infected individuals), ${ }^{24-26}$ and CXCR5 (which is expressed on Tfh or memorylike CD4 T cells) on ESAT-6/CFP-10-specific CD4 T cells in several different tissues at the time of necropsy (Figure $\mathbf{4 c , d}$ ). In each tissue, we observed the same hierarchy of chemokine receptor expression on Mtb-specific CD4 T cells: CXCR3 > CCR5 > CCR6 with little or no CX3CR1 and CXCR5. Therefore, Mtb-specific CD4 T cells in acutely infected rhesus macaques largely express the Th1 chemokine receptors CXCR3 and CCR5, which is consistent with previous reports in macaques. ${ }^{27}$

Expression of co-inhibitory molecules PD-1 and CTLA-4 on Mtb-specific CD4 T cells is increased at sites of infection In the mouse model of tuberculosis, the co-inhibitory receptors PD-1 and CTLA-4 have been found to be selectively expressed by Mtb-specific CD4 $\mathrm{T}$ cells that have migrated into the lung tissue parenchyma. ${ }^{7,828}$ In fact, in some studies PD-1 has been used as a surrogate marker for the host-protective subset of CD4 T cells in Mtb-infected mice. ${ }^{8,15}$ Therefore, we examined the expression of PD- 1 and CTLA- 4 on Mtb-specific CD4 T cells in various tissues of infected macaques. We found that the gMFI of PD-1 expression on ESAT-6/CFP10-specific CD4 T cells was higher in granulomas compared with the blood, spleen, and lymph nodes (Figure 5a,b). Interestingly, we also noted that PD-1 expression on ESAT-6/CFP-10-specific and PPD-specific CD4 T cells was higher compared with TB10.4specific CD4 $\mathrm{T}$ cells when we compared samples for which we had all three stimulation conditions (Figure 5c,d). CTLA-4 expression was lowest on ESAT-6/CFP-10-specific CD4 T cells in the blood, spleen, and BAL, and highest on cells in the lymph nodes, instillation site lesions, and granulomas (Figure 5e,f). Interestingly, when combining samples isolated from different tissues, we observed a significant correlation between the gMFI of PD-1 on Mtb-specific CD4 T cells and the fraction of cells 
a
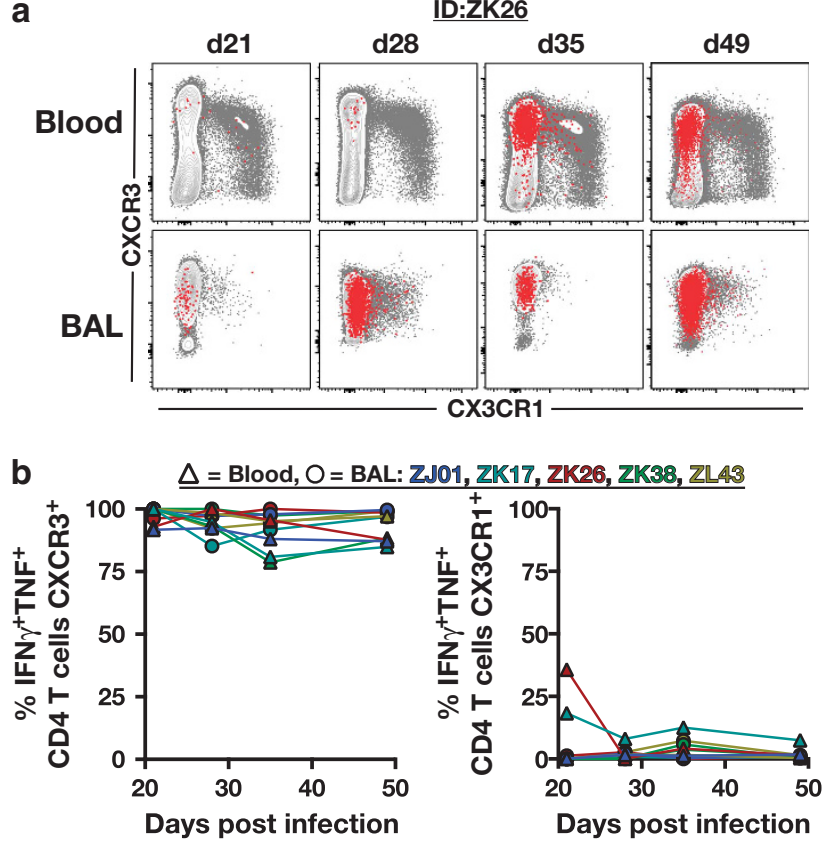

C

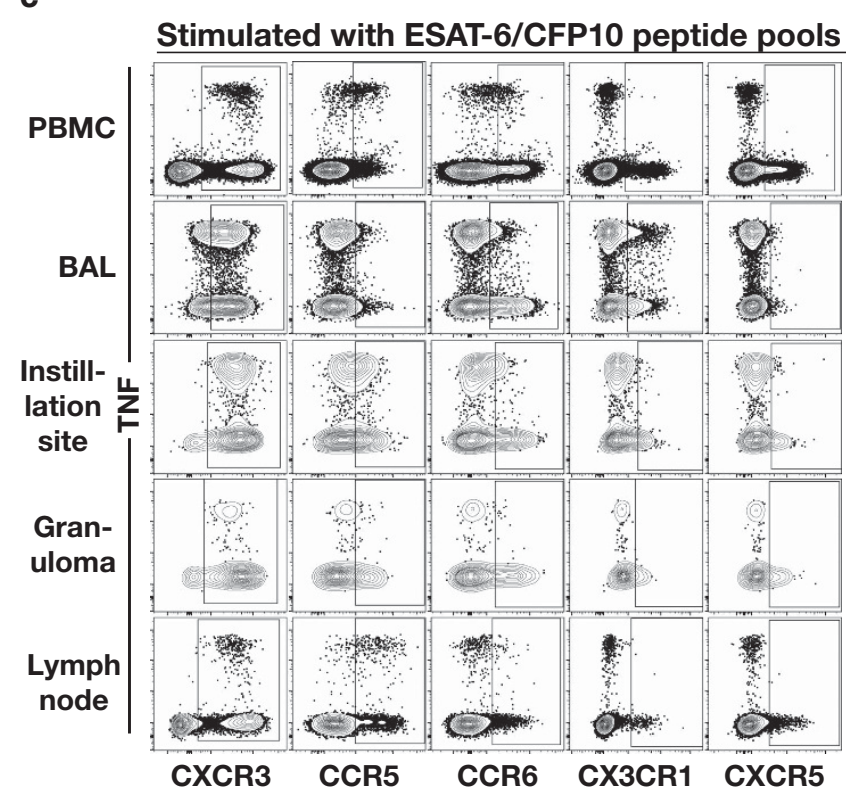

d

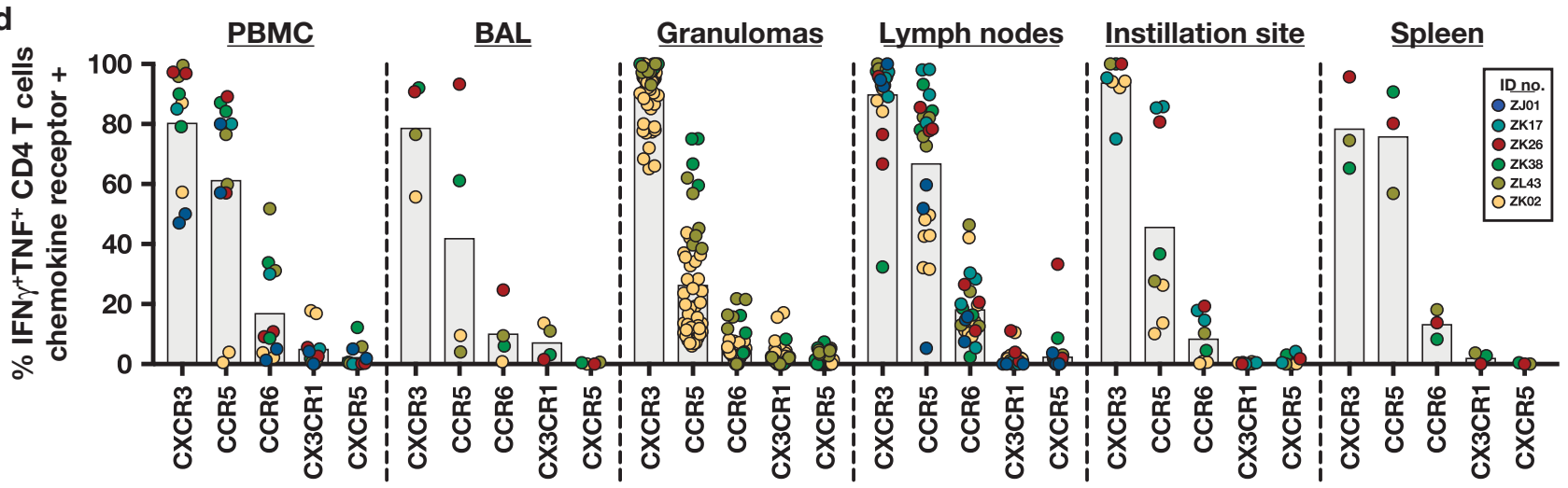

Figure 4 Ag-specific CD4 T cells do not differentiate into CX3CR1 ${ }^{+} \mathrm{CXCR} 3^{-}$terminal effectors in primary Mtb infection of rhesus macaques. (a,b) Example fluorescence-activated cell sorting (FACS) plots (a) and summary quantifications (b) of CXCR3 and CX3CR1 expression on bulk (gray) or ESAT-6/CFP-10-specific (red dots) CD4 T cells from the blood and bronchoalveolar lavage (BAL). Overlaid Ag-specific CD4 T cells are gated on $\mathrm{TNF}^{+} \mathrm{IFN} \gamma^{+}$cells following stimulation with peptide pools. (c,d) Example FACS plots (c) and summary quantifications (d) of chemokine receptor expression on ESAT-6/CFP-10-specific CD4 T cells from various tissues on day 60 post infection.

that expressed CTLA-4 (Figure 5g). Therefore, Mtb-specific CD4 T cells express relatively high amounts of PD-1 and CTLA-4 at the site of Mtb infection.

\section{Poor penetration of CD4 T cells into the infected macrophage core of granulomas}

It has been previously shown that the majority of lymphocytes in granulomas are present in a peripheral ring circumscribing the lesion, often referred to as the lymphocyte cuff, but CD4 T cells themselves have not been directly addressed. ${ }^{17,29,30}$ Therefore, we sought to quantify the localization of CD4 T cells into different regions of the granuloma. To do so, immediately after resection we embedded live granulomas in agarose and cut $300-400 \mu \mathrm{m}$-thick sections that were then stained and imaged by confocal microscopy in the BSL3 without fixation (Figure 6a). We identified several distinct regions working from the peripheral edge inward (Figure $6 \mathbf{b}$ ). The outermost region was defined as the lymphocyte cuff, which is rich in $\mathrm{CD} 4{ }^{+} \mathrm{T}$ cells and interspersed with $\mathrm{CD}_{11 \mathrm{~b}}{ }^{+}$cells. We repeatedly observed a gap in $\mathrm{CD} 11 \mathrm{~b}$ staining between the lymphocyte cuff and $\mathrm{CD} 11 \mathrm{~b}^{+}$core. This region may represent an area of fibrosis or epithelioid macrophages. However, we have not directly demonstrated either, so here we will refer to the non-staining region between the lymphocyte cuff and the core as the granuloma lacunar zone. We also noticed that CD4 $\mathrm{T}$ cells were often found lining up at the edge of the inner core, so we identified the boundary of the $\mathrm{CD}_{11} \mathrm{~b}^{+}$core as a distinct region. Lastly, we identified the $\mathrm{CD} 11 \mathrm{~b}^{+}$myeloid cell rich core. We calculated the percentage of total granuloma CD4 T cells that are present in each region of the granuloma to measure their distribution. As expected, the vast majority of CD4 T cells were in the outer regions of the granuloma and the percentage of CD4 $\mathrm{T}$ cells in each region fell progressively towards the center of the granuloma (Figure 6c). On either extreme, 65\% 
a

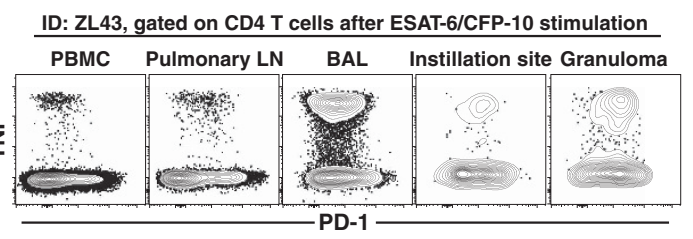

d

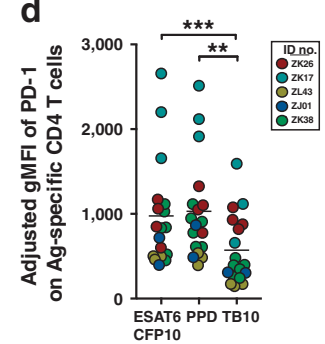

b

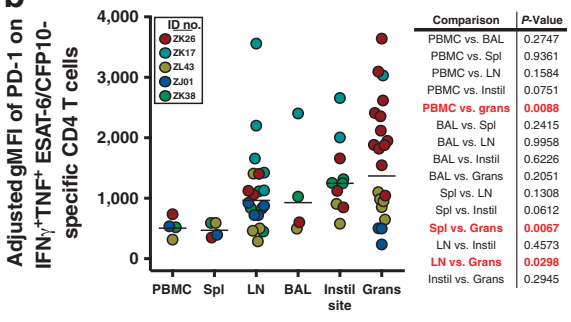

f

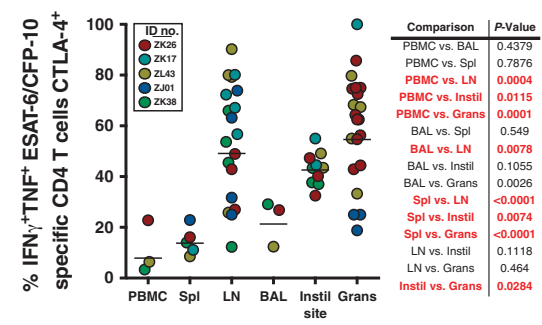

C

ID: ZK38, pulmonary LN

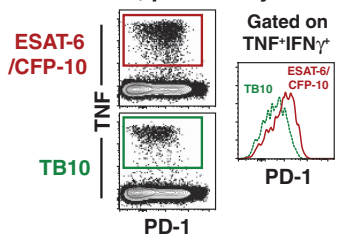

g

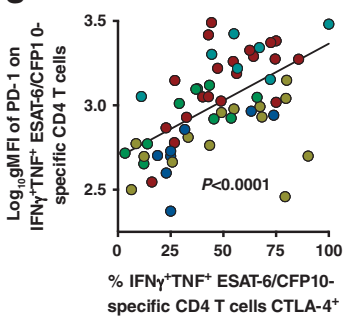

Figure 5 PD-1 and CTLA-4 expression on Mtb-specific CD4 T cells are elevated at sites of bacterial replication. (a,b) Example fluorescence-activated cell sorting (FACS) plots (a) and quantifications (b) of PD-1 expression in ESAT-6/CFP-10-specific CD4 T cells in different tissues of Mtb-infected rhesus macaques. In order to better compare across different necropsy days, the adjusted gMFI of PD-1 expression was calculated as the gMFI of PD-1 on Ag-specific CD4 T cells minus the gMFI of PD-1 on PD-1 negative lymph node CD4 T cells from the matched animal. $P$-values for one-way analysis of variance (ANOVA) with Fisher's least significant different (LSD) test for multiple comparisons shown to the right of the graph with significant differences highlighted in red. (c,d) PD-1 expression was compared on ESAT-6/CFP-10-, PPD-, and TB10.4-specific CD4 T cells. Example FACS plots are shown in $\mathbf{c}$ and summary graphs in $\mathbf{d}$. Data from various tissues at necropsy are pooled and only samples where data from all three stimulation conditions were available are included for $t$-test comparison. Example FACS plots (e) and summary graphs (f) of the percentage of ESAT-6/CFP-10-specific CD4 T cells that expressed intracellular CTLA-4 following stimulation. $P$-values for one-way ANOVA with Fisher's LSD test for multiple comparisons shown to the right of the graph with significant differences highlighted in red. (g) Correlation of the adjusted gMFI of PD-1 and the percent CTLA-4 ${ }^{+}$in ESAT-6/CFP-10specific CD4 T cells. Data from various tissues at necropsy are pooled. ${ }^{\star \star \star \star} P<0.0001,{ }^{\star \star \star} P<0.001$, and ${ }^{\star} P<0.05$.
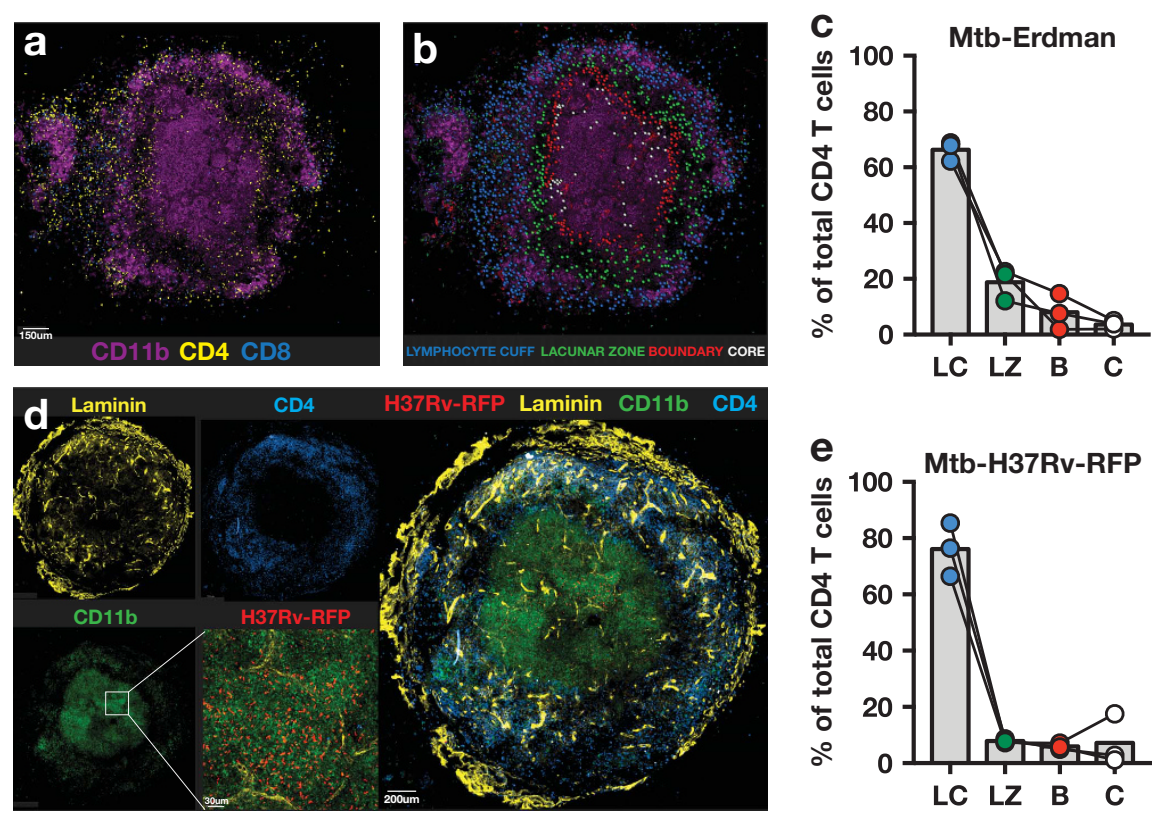

Figure 6 CD4 T cells poorly localize to the infected macrophage-rich center of granulomas. (a-c) Individually resected pulmonary granulomas from rhesus macaques infected with Mtb-Erdman were thick-sectioned, stained with an antibody cocktail and imaged for the presence and localization of CD4 $\mathrm{T}$ cells. (a) Example image showing the location of $\mathrm{CD}^{+}{ }^{+}$and $\mathrm{CD} 8^{+}$cells in relation to CD11b-expressing cells. (b) Four different regions of the granuloma were identified as described in the text and CD4 T cells in each region highlighted in a different color: lymphocyte cuff (blue), lacunar zone between the lymphocyte cuff, and the macrophage core (green), boundary of the macrophage-rich core (red), and the macrophage-rich core (white). (c) Quantification of the percent of $\mathrm{CD} 4^{+}$cells that are in each region of the granuloma. Data shown are from three separate granulomas. (d,e) Two rhesus macaques were infected with $\sim 8$ cfu of Mtb-H37Rv-RFP and granulomas were resected 6-7 weeks post infection for analysis of CD4 T-cell localization as above. (d) Individual staining of laminin, CD11b, CD4, and RFP-Mtb on the left with a merged tile on the right. (e) Quantification of the percent of CD4 ${ }^{+}$ cells that are in each region. Three separate granulomas from these two Mtb-H37Rv-RFP-infected animals were analyzed. 
of CD4 T cells localized into the lymphocyte cuff and only 3\% of the total CD4 $\mathrm{T}$ cells were in the myeoid cell core, presumably where the bacteria are located.

We next sought to confirm these observations and examine the distribution of CD4 T cells in granulomas from animals infected with a less virulent strain of Mtb, H37Rv. Two additional rhesus macaques were infected with $\sim 8 \mathrm{CFUs}$ of $\mathrm{H} 37 \mathrm{Rv}$-RFP, which also allowed us to visualize the bacteria. In contrast to the MtbErdman infected animals, these macaques had neither developed large consolidation-like lesions at the site of instillation nor shown any signs of disease by the time of necropsy (day 42 and 49 ). We also observed far fewer granulomas in the H37Rv-RFP-infected animals ( 5-7 granulomas in H37Rv-RFP versus $\sim 30-80$ in Erdman). The reduced disease may have resulted from the decreased virulence of $\mathrm{H} 37 \mathrm{Rv}$ compared with Erdman in nonhuman primates (NHP), ${ }^{31}$ bacterial attenuation introduced by the red fluorescent protein (RFP) reporter, and/or the lack of repeated BAL sampling during infection. We stained the granulomas for laminin to identify blood vessels, CD4, and CD11b (Figure 6d). Similar to the Mtb-Erdman granulomas, we found that $\sim 75 \%$ of CD4 T cells were in the lymphocyte cuff, with only $\sim 1-17 \%$ CD4 $\mathrm{T}$ cells having migrated past the border of the central myeloid cell core where the bacteria are located (Figure 6e and Supplementary Movie online). Combining the data sets from all of the six granulomas imaged, only $4.6 \pm 2.2 \%$ of all CD4 T cells in the granuloma have entered the central region. Therefore, the vast majority of CD4 T cells inside of granulomas are not in contact with Mtb-infected cells.

\section{Intravascular staining to measure migration of Ag-specific effector CD4 T cells out of the blood into pulmonary lesions in Mtb-infected rhesus macaques}

Mtb-specific effector CD4 T cells in rhesus macaques display a phenotype similar to the subset of CD4 T cells in mice that is able to extravasate out of the blood into the lung tissue $\left(\mathrm{CXCR} 3{ }^{+} \mathrm{PD}-1^{+}\right.$CTLA $-4^{+}$CX3CR $\left.1^{-}\right)$. However, at the same time, the CD4 $\mathrm{T}$ cells are almost entirely localized to the outer zone of the granuloma, which may be due to poor extravasation out of vessels. Therefore, we next sought to directly quantify the parenchymal versus intravascular localization of Mtb-specific effector CD4 T cells.

In order to quantify the fraction of CD4 $\mathrm{T}$ cells localizing within blood vessels, we adapted the flow cytometry-based intravascular staining approach commonly applied in mouse studies for use in macaques. ${ }^{32}$ In short, anesthetized macaques were intravenously infused with a biotinylated anti-macaque $\mathrm{CD} 45 \mathrm{mAb}$, and $\sim 10 \mathrm{~min}$ later the animals were exsanguinated, sacrificed, and necropsied. Initially, we tested the technique on an uninfected macaque (Figure 7a). Blood taken immediately before infusion was completely negative for iv stain, whereas blood taken just before euthanasia was uniformly positive. $\mathrm{CD} 4 \mathrm{~T}$ cells isolated from the airways were completely iv stain negative, whereas lung tissue CD4 T cells showed the typical iv stain-positive and -negative populations observed in murine studies. Moreover, iv stain-negative CD4 T cells in the lung were greatly enriched for CD69, a marker for tissue resident $\mathrm{T}$ cells (Figure 7a). The technique also identified intravascular and parenchymal CD4 T cells in other tissues such as the lymph node and brain, and again $\mathrm{CD} 69^{+}$cells were greatly enriched in the iv stain-negative populations (Figure 7a). Moreover, we found that CX3CR1 ${ }^{+} \mathrm{CD} 4 \mathrm{~T}$ cells obtained from lung tissue in an uninfected macaque were uniformly iv stain positive (Figure $7 \mathbf{b}$ ). Collectively, these results indicate that the intravascular staining approach can be used to quantify lymphocyte migration into tissues in rhesus macaques and $\mathrm{CX} 3 \mathrm{CR} 1^{+}$terminal effector $\mathrm{CD} 4 \mathrm{~T}$ cells localize predominately within lung blood vessels as we have previously reported in mice.

We used the intravascular staining approach to quantify the localization of Mtb-specific CD4 T cells within various tissues of infected rhesus macaques $\sim 2$ months after infection. As expected, ESAT-6/CFP-10-specific CD4 T cells were iv stain positive in the blood and negative in the BAL (Figure $7 \mathbf{c}, \mathbf{d}$ ). Interestingly, we observed that in instillation site lesions and lung granulomas, there were little to no intravascular Mtbspecific effector CD4 $\mathrm{T}$ cells. These results are consistent with the lack of CX3CR1 ${ }^{+}$terminal effector $\mathrm{CD} 4 \mathrm{~T}$ cells in rhesus macaques, but contrast dramatically with mice. Collectively, these results show that in rhesus macaques, the vast majority of primary Mtb-specific effector CD4 $\mathrm{T}$ cells are efficient lunghoming cells, and very few adopt the intravascular terminal effector phenotype. Moreover, these data indicate that poor localization of effector $\mathrm{CD} 4 \mathrm{~T}$ cells into the central region of the granuloma is not due to defects in transendothelial diapedesis.

\section{DISCUSSION}

Data from mice have shown that the protective capacity of Mtbspecific Th1 cells is tightly linked to T-cell differentiation state, largely due to changes in lung-homing ability that occur as Th1 cells progressively differentiate. Although interleukin-12/Tbet-driven CD4 T-cell polarization is required for Th1 cell generation and secretion of IFN $\gamma$, extensive differentiation leads to the generation of terminal effector cells that neither migrate into the lungs nor mediate control of Mtb infection in adoptive transfer experiments. Therefore, $\mathrm{T}$ cells with intermediate levels of Th1 differentiation display the best protective capacity in murine studies. Here we tested these hypotheses arising from basic murine model studies in non-human primates by examining the differentiation and lung-homing of effector CD4 T cells following primary Mtb infection. We found that, unlike mice, the Mtb-specific CD4 T cells in this group of rhesus macaques did not undergo terminal differentiation into a CX3CR $1^{+} \mathrm{CXCR} 3{ }^{\text {low }}$ phenotype during the primary clonal burst. Instead, the responding effector CD4 T cells display markers consistent with parenchymal cells from mouse studies including: $\mathrm{CXCR} 3^{+} \mathrm{PD}-1^{+} \mathrm{CTLA}-4^{+}$. Moreover, using the intravascular staining technique, we find no accumulation of intravascular effector CD4 $\mathrm{T}$ cells in rhesus macaques. We did observe a small amount of CX3CR1 expression on Mtb-specific CD4 T cells, but only at a lower MFI and on cells that had not downregulated CXCR3. This is a phenotype consistent with a recent study showing that 
a

Gated on CD4 T cells in uninfected rhesus macaque

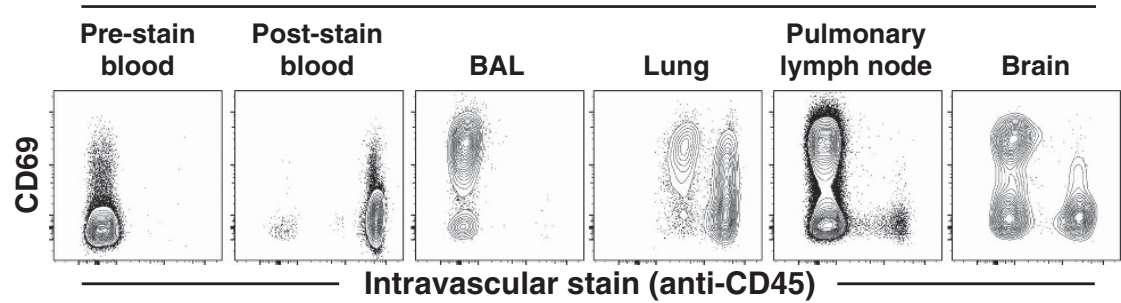

b
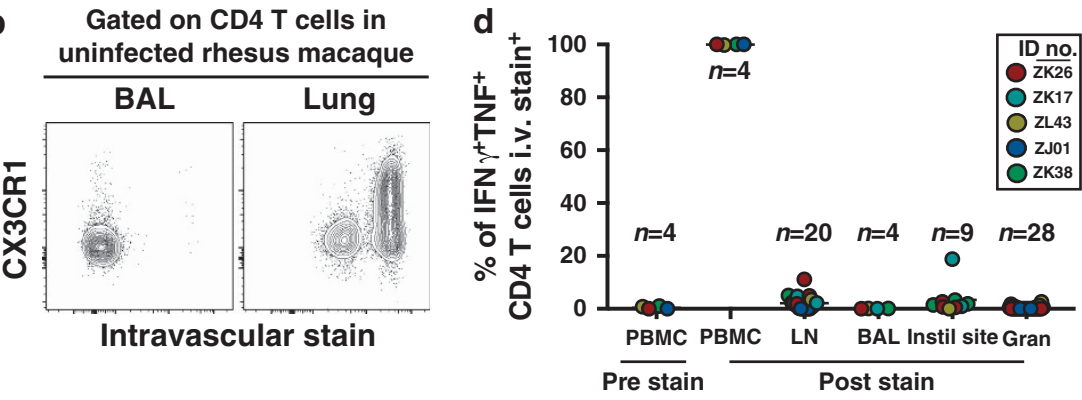

C Gated on CD4 T cells stimulated with ESAT-6/CFP10 peptide pools

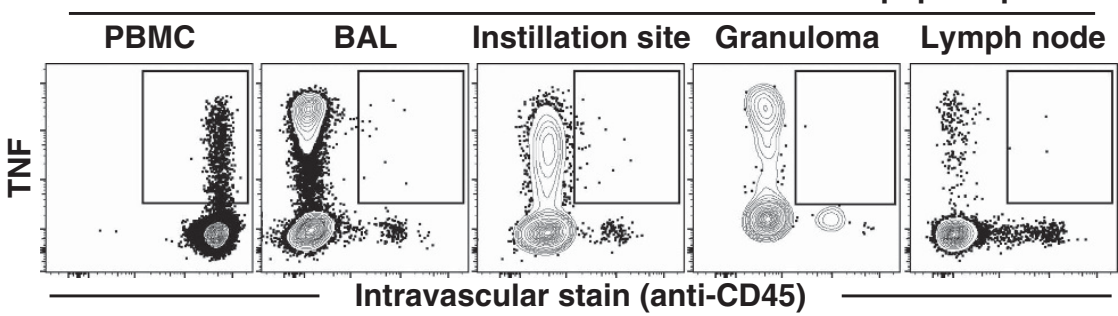

Figure 7 Ag-specific CD4 T cells efficiently extravasate out of the lung blood vasculature into parenchymal pulmonary lesions. Just before euthanasia and necropsy, $\alpha \mathrm{CD} 45$-biotin mAb was intravenously injected into the animals to allow for the detection of intravascular and parenchymal lymphocytes by flow cytometry. (a) Staining for the iv injected $\alpha \mathrm{CD} 45 \mathrm{mAb}$ and CD69 are shown on CD4 T cells in various tissues from an uninfected rhesus macaque. (b) CX3CR1 and iv $\alpha$ CD45 stain on CD4 T cells from the BALf and lung tissue of an uninfected rhesus macaque. (c) Example fluorescence-activated cell sorting (FACS) plots of intravascular staining on ESAT-6/CFP-10-specific CD4 T cells from different tissues of an Mtb-Erdman infected rhesus macaque. (d) Summary graphs of the percent of ESAT-6/CFP-10-specific CD4 T cells that are positive for the intravascular CD45 stain from various tissues of MtbErdman infected rhesus macaques. Ag-specific CD4 T cells were identified by gating on $\mathrm{TNF}^{+} \mathrm{IFN} \gamma^{+}$cells following restimulation with peptide pools.

CX3CR1 ${ }^{\text {dim }}$ cells are a unique subset of $\mathrm{T}$ cells that are more similar in functional properties to less-differentiated cells, including tissue homing. ${ }^{33}$ Therefore, these data indicate that the great majority of macaque $\mathrm{CD} 4 \mathrm{~T}$ cells do not "overpolarize" and become terminal effectors that cannot enter the lung, as is observed in the mouse.

We should point out, however, that terminal differentiation may occur in other settings of Mtb infection, such as during chronic tuberculosis or after priming macaques with different vaccine vectors. Our data do confirm that CX3CR $1^{+}$terminal effector CD4 T cells in NHP also poorly migrate out of the blood vasculature and into the lung, so even if terminal effectors cells are not generated after natural infection, vaccines that selectively induce CX3CR1 ${ }^{+}$Mtb-specific CD4 T cells may be less desirable. Monitoring CD4 T cells for the expression of CXCR3 and CX3CR1 may give insight into the lung-homing capacity of the vaccine-generated $\mathrm{T}$ cells in future studies.

We observed other informative differences in the quality of Mtb-specific CD4 T cells in macaques compared with mice. In particular, we detected CCR6 expression (normally associated with Th17 polarized cells) on a fraction of Mtb-specific
$\mathrm{CXCR}^{+}$IFN $\gamma$-producing CD4 T cells, which is not observed in mice. Human Mtb-specific CD4 T cells have been reported several times previously to co-express CXCR3 and CCR6, thereby bearing markers of both Th1 and Th17 cells, but primarily produce IFN $\gamma$ and not interleukin-17A. . $^{2,25,34,35}$ Macaque CD4 T cells seem to more closely resemble human CD4 T cells in this respect, although we only observed CCR6 on a subset of CXCR3 ${ }^{+} \mathrm{CD} 4 \mathrm{~T}$ cells in macaques as compared with a majority of $\mathrm{CXCR} 3^{+}$cells as has been reported in humans. $\mathrm{CXCR}^{+}{ }^{+} \mathrm{CCR}_{6}{ }^{+} \mathrm{CD} 4 \mathrm{~T}$ cells, sometimes referred to as Th1 ${ }^{*}$, Th1/Th17, or Th17.1 cells, have been observed in several different clinical conditions including multiple sclerosis ${ }^{36}$ and sarcoidosis, ${ }^{37}$ and found to be dependent on interleukin- $1 \beta$ for their generation. ${ }^{38}$ Interestingly, $\mathrm{CXCR} 3{ }^{+} \mathrm{CCR} 6{ }^{+} \mathrm{CD} 4 \mathrm{~T}$ cells in individuals with inborn errors in $R O R C$ have defective IFN $\gamma$ production and these individuals are unable to control mycobacterial infections. ${ }^{39}$ Therefore, our data are consistent with previous data showing that $\mathrm{CD} 4 \mathrm{~T}$ cell polarization in human Mtb infection primarily generates mixtures of less-differentiated Th1 as well as Th1* cells rather than the highly focused and extensive Th1 differentiation seen in 
C57BL/6 mice. Collectively, these data show that defects in CD4 $\mathrm{T}$-cell migration into granulomas are unlikely to contribute to the poor control of Mtb infection.

However, although we show that CD4 T cells in Mtb-infected rhesus macaques efficiently migrate out of the blood vessels into the pulmonary lesions, here we also confirm that $\mathrm{CD} 4 \mathrm{~T}$ cells progressively decrease in abundance approaching the infected macrophage-rich center of the granuloma. This was true even in granulomas at relatively early time points. Given the murine model data indicating that $\mathrm{CD} 4 \mathrm{~T}$ cells must interact with macrophages to suppress the growth of Mtb infection, this defect in intralesional positioning may pose a major barrier to CD4 $\mathrm{T}$-cell-mediated control of Mtb infection. Indeed, this raises the basic question of what are the mechanisms that regulate the penetration of CD4 $\mathrm{T}$ cells into the infected granuloma core. Assuming that the small subset of $\mathrm{CD} 4 \mathrm{~T}$ cells that does enter the central core are responsible for supplying help to infected myeloid cells, a more practical implication is that the number of Ag-specific CD4 T cells in a granuloma does not reflect the actual number of protective $\mathrm{T}$ cells. Regardless of the total number of Ag-specific CD4 T cells inside of granulomas, the actual local effector:target ratio may be far below what is required for proper macrophage help and bacterial clearance. From this perspective, data obtained from the study of peripheral blood cells or even BAL cells seem difficult to interpret.

In all, the data presented here have failed to support the original hypothesis developed in mouse models and instead highlight the importance of a long-recognized but relatively unexplored aspect of CD4 T-cell behavior in tuberculosis. Although it has been known for decades that lymphocytes are rare in central regions of granulomas, almost nothing is known about the signals governing $\mathrm{CD} 4 \mathrm{~T}$-cell entry into granulomas or the mechanisms regulating how they distribute themselves throughout the granuloma after extravasation across the blood vascular endothelium. A better understanding of the basic immunological mechanisms that regulate leukocyte trafficking within granulomas may give insight into the underlying defects in host resistance to Mtb infection.

\section{METHODS}

Animals, infections, and CFU quantification. Six healthy, PPD skin test negative rhesus macaques originally from the Morgan Island NIH breeding colony were chosen for this study and housed in a Biosafety Level 3 vivarium. All six animals were exposed to an ultra-low dose $(<1 \mathrm{cfu})$ of Mtb-Erdman strain via bronchoscopic instillation into the lower right lung lobe. Dose was confirmed by plating of inoculum onto 7H11 agar plates, which grew zero colonies. One animal (ZK02) became infected as confirmed by PPD skin test, the presence of an antigen-specific T-cell response, and the presence of severe disease by PET/CT scan. The remaining five animals were negative for the presence of Mtb infection as confirmed by those same parameters. Those five were then re-exposed to an ultra-low dose $(6-10 \mathrm{cfu})$ via the same method. The presence of infection after the second exposure was confirmed. All CFU measurements were generated by serial dilutions on 7H11 agar plates supplemented with OADC (Difco, Detroit, MI). For the H37Rv-RFP infection, the animals were bronchoscopically exposed to $\sim 8$ CFUs.

The animals were housed in non-human primate bio-containment racks and maintained in accordance with the Animal Welfare Act, the
Guide for the Care and Use of Laboratory Animals, and other Federal statutes and regulations relating to animals, in a fully Association for Assessment and Accrediation of Laboratory Animal Care (AAALAC)accredited facility. Housing was in accord with the National Institute of Allergy and Infectious Diseases Division of Intramural Research Animal Program Policy on Social Housing of Non-Human Primates.

The animals were fed a high fiber commercial diet that contains $20 \%$ protein twice a day (2050 Harlan Teklad Global Primate Diet, Envigo, Huntingdon, UK). Fruit supplements (various fresh fruits) are given at least three times per week. Animals receive water and PRANG oral rehydrator (Bio-Serv, Flemington, NJ) daily in water bottles with sipper tubes. Daily environmental enrichment is provided in the form of various hanging devices, manipulanda, and a variety of food items such as PRIMA treats (Bio-Serv, Flemington, NJ), peanut butter, Nutrablocks (Bio-Serv, Flemington, NJ), peanuts, and popsicles, in accord with National Institute of Allergy and Infectious Diseases Division of Intramural Research Comparative Medicine Branch Standard Operating Procedures and animal facility practices.

All procedures were performed utilizing appropriate anesthetics as listed in the National Institute of Allergy and Infectious Diseases Division of Intramural Research Animal Care and Use Committee approved animal study proposal LPD 25E. Euthanasia methods were consistent with the American Veterinary Medical Association Guidelines on Euthanasia and the endpoint criteria listed in the National Institute of Allergy and Infectious Diseases Division of Intramural Research Animal Care and Use Committee approved animal study proposal LPD 25E.

Sample collection. Blood and BAL collections were performed weekly through the course of infection. An aliquot was taken for CFU measurement. Subsequently, peripheral blood mononuclear cells were isolated using Ficoll-paque PLUS gradient separation (GE Healthcare Biosciences, Pittsburgh, PA). For BAL collections, tubing was inserted into the trachea with assistance using a laryngoscope, whereupon saline was instilled and immediately aspirated. An aliquot was taken for CFU and cells were isolated for flow cytometry analysis.

Intravascular stain. Before injection and euthanasia, blood was drawn as a negative stain control. Fifty to $100 \mu \mathrm{g} \mathrm{kg}^{-1}$ of $\alpha \mathrm{CD} 45$-biotin (clone MB4-6D6, Miltenyi Biotec, San Diego, CA) was diluted into $20 \mathrm{ml}$ of sterile saline and injected into the saphenous vein. After $10 \mathrm{~min}$, the animal was exsanguinated and humanely euthanized. Lymphocytes were isolated from the blood as described above. Cells were then stained with a fluorochrome-labeled streptavidin during the flow cytometry staining protocol and analyzed.

Lymphocyte isolation, in vitro stimulation, and flow cytometry. Upon resection, lungs and instillation site lesions were minced and enzymatically digested using a gentleMACS dissociator (Miltenyi Biotec) in RPMI-1640 medium supplemented with $1 \mathrm{mg} \mathrm{ml}^{-1}$ Collagenase D (Roche-Diagnostics, Indianapolis, IN), $1 \mathrm{mg} \mathrm{ml}^{-1}$ hyaluronidase, and $50 \mathrm{U} \mathrm{ml}^{-1}$ DNase 1 (both from Sigma-Alrich, St Louis, MO). Homogenates were then passed through a $100 \mu \mathrm{m}$ cell strainer and enriched for lymphocytes using a 25\%/50\% Percoll density gradient centrifugation. Granulomas were simply mashed through a $100 \mu \mathrm{m}$ cell strainer and cells collected for analysis.

For T-cell stimulations, cells were incubated for $6 \mathrm{~h}$ at $37^{\circ} \mathrm{C}$ in X-Vivo 15 media supplemented with $10 \%$ fetal calf serum and brefeldin A, and monensin along with $1 \mu \mathrm{g} \mathrm{ml}^{-1}$ ESAT-6/CFP-10 peptide pools, $1 \mu \mathrm{g} \mathrm{ml}^{-1}$ TB10.4 (all from BEI Resources, Manassas, VA), and $10 \mu \mathrm{g} \mathrm{ml}^{-1}$ PPD (Statens Serum Institut, Copenhagen, Denmark). Cells were then stained with various combinations of the following fluorochrome-labeled antibodies: CD3 (SP34-2), CD4 (OKT4), CD8 (RPA-T8), CCR5 (3A9), CCR6 (11A9), CXCR3 (G025H7), CXCR5 (MU5UBEE), CX3CR1 (K0124E1), PD-1 (EH12.2H7), CTLA-4 (14D3), tumor necrosis factor (MAB11), IFN $\gamma$ (4S.B3), CD69 (FN50), and Fixable Viability Dye eFluor 780, all purchased from BioLegend, 
eBioscience (San Diego, CA), or BD Biosciences (San Jose, CA). All samples were acquired on a BD LSRfortessa flow cytometer and analyzed using FlowJo software (Version 10.0.8, Tree Star, Ashland, OR). Supplementary Figure 1 shows a typical gating strategy.

PET/CT scans and analysis. All PET/CT scans were performed using a hybrid system that includes a micro-PET Focus 220 scanner (Siemens Molecular Solutions, Knoxville, TN) and an eight-slice helical CT scanner (Neurologica, Danvers, MA). Calculations for a standardized uptake value ratio (SUVR) were made by dividing the total metabolic activity (SUV>2.3) of either the entire lung or individual instillation site lesions and pulmonary lymph nodes by the cylinder muscle value to normalize for scan differences, as described previously. ${ }^{40}$ All calculations were made using OsiriX MD (version 8.0.2, Pixmeo, Switzerland).

Confocal microscopy of live granuloma sections. Granulomas were kept on ice, in $1 \%$ fetal bovine serum in phosphate-buffered saline. Preheated 2\% agarose (Lonza, Basel, Switzerland) in RPMI was chilled to $37^{\circ} \mathrm{C}$ and immediately poured over the granulomas in a Petri dish and placed on ice. Upon agarose gel polymerization, agarose gel was sliced into $300-350 \mu \mathrm{m}$ sections using Leica VT1000 S Vibrating Blade Microtome (Leica Microsystems, Wetzlar, Germany) at speed 5, in icecold phosphate-buffered saline. Tissue sections were stained with fluorescently labeled anti-CD4, -CD11b, -CD8, -CD20, or -CD31 antibody of choice (eBioscience) for $2 \mathrm{~h}$ on ice. After staining sections were washed three times and cultured in complete lymphocyte medium (Phenol Red-free RPMI supplemented with 10\% fetal bovine serum, 10\% rhesus plasma, $25 \mathrm{~mm}$ HEPES, $50 \mu \mathrm{M} \beta$-ME, $1 \%$ Pen/Strep/L-Glu and 1\% sodium pyruvate) in humidified incubator at $37^{\circ} \mathrm{C}$. Tissue sections were allowed complete recovery in the incubator for $12 \mathrm{~h}$ before imaging. Sections were imaged using Leica SP5 inverted 5 channel confocal microscope equipped with an Environmental Chamber (NIH Division of Scientific Equipment and Instrumentation Services, Bethesda, MD) to maintain $37^{\circ} \mathrm{C}$ and $5 \% \mathrm{CO}_{2}$. Microscope configuration was set up for four-dimensional analysis $(x, y, z, t)$ of cell segregation and migration within tissue sections. Diode laser for $405 \mathrm{~nm}$ excitation; Argon laser for 488 and $514 \mathrm{~nm}$ excitation, DPSS laser for 561, and HeNe lasers for 594 and $633 \mathrm{~nm}$ excitation wavelengths were tuned to minimal power (between 1 and 5\%). Z-stacks of images were collected $(10-50 \mu \mathrm{m})$. Mosaic images of complete granuloma sections were generated by acquiring multiple $Z$-stacks using motorized stage to cover the whole section area and assembled into a tiled image using LAS X (Leica Microsystems) software. Post-acquisition images were processed using Imaris 8.4.1 (Bitplane) software for quantification and visualization. Localizations of the cells in granulomas were identified based on fluorescence intensity using spot function of Imaris and divided into four different regions based on localization using surface three-dimensional reconstruction and channel masking method.

Statistical analysis. All statistical analysis was performed in Prism (GraphPad Software, La Jolla, CA). Two-tailed $t$-test, paired $t$-test, one-way analysis of variance with Fisher's least significant different test for multiple comparisons were used in the analysis.

SUPPLEMENTARY MATERIAL is linked to the online version of the paper at http://www.nature.com/mi

\section{ACKNOWLEDGMENTS}

We are grateful to Nannan Zhu and the NIAID aBSL3 facility technicians for laboratory and non-human primate technical support. We thank Dr Richard Herbert and his team for help with the establishment of bronchoscopic infection and development of the intravascular stain approach in NHP. We thank Robert Seder, Patricia Darrah, and Mario Roederer for sharing unpublished data on the NHP iv stain technique. We thank JoAnne Flynn, Charles Scanga, and Alex White for instruction on low-dose Mtb infections and PET/CTanalysis. We are also thankful to Sivaranjani Namasivayam and Katrin Mayer-Barber for help with experiments in the BSL3. This work was supported by the Intramural Research Program of NIAID/NIH.

\section{AUTHOR CONTRIBUTIONS}

K.D.K. and D.L.B. conceived the study, analyzed data, and wrote the manuscript. K.D.K., M.A.S., S.S., and O.K. performed experiments. M.S., D.S., and C.E.B.III performed and provided expertise on PET-CT. R.M., T.W.K., I.M., D.W., and L.V. performed necropsies. J.K. analyzed imaging data. R.M. and T.W.K. performed the bronchoscopic infections and provided veterinary care for infected NHP under aBSL3 housing.

\section{DISCLOSURE}

The authors declare no conflict of interest.

c) 2018 Society for Mucosal Immunology

\section{REFERENCES}

1. Orme, I.M. The kinetics of emergence and loss of mediator T lymphocytes acquired in response to infection with Mycobacterium tuberculosis. J. Immunol. 138, 293-298 (1987).

2. Muller, I., Cobbold, S.P., Waldmann, H. \& Kaufmann, S.H. Impaired resistance to Mycobacterium tuberculosis infection after selective in vivo depletion of L3T4 + and Lyt-2 + T cells. Infect. Immun. 55, 2037-2041 (1987).

3. Lin, P.L. et al. CD4 T cell depletion exacerbates acute Mycobacterium tuberculosis while reactivation of latent infection is dependent on severity of tissue depletion in cynomolgus macaques. AIDS Res. Hum. Retroviruses 28, 1693-1702 (2012).

4. Yao, S., Huang, D., Chen, C.Y., Halliday, L., Wang, R.C. \& Chen, Z.W. CD4 + T cells contain early extrapulmonary tuberculosis (TB) dissemination and rapid TB progression and sustain multieffector functions of CD8 + Tand CD3- lymphocytes: mechanisms of CD4 + T cell immunity. J. Immunol. 192, 2120-2132 (2014).

5. Srivastava, S. \& Ernst, J.D. Cutting edge: direct recognition of infected cells by CD4 $\mathrm{T}$ cells is required for control of intracellular Mycobacterium tuberculosis in vivo. J. Immunol. 191, 1016-1020 (2013).

6. Sakai, S. et al. CD4 Tcell-derived IFN-gamma plays a minimal role in control of pulmonary Mycobacterium tuberculosis infection and must be actively repressed by PD-1 to prevent lethal disease. PLoS Pathog. 12, e1005667 (2016).

7. Sakai, S. et al. Cutting edge: control of Mycobacterium tuberculosis infection by a subset of lung parenchyma-homing CD4 T cells. J. Immunol. 192, 2965-2969 (2014).

8. Moguche, A.O. et al. ICOS and Bcl6-dependent pathways maintain a CD4 T cell population with memory-like properties during tuberculosis. J. Exp. Med. 212, 715-728 (2015).

9. Sallin, M.A., Sakai, S., Kauffman, K.D., Young, H.A., Zhu, J. \& Barber, D.L. Th1 differentiation drives the accumulation of intravascular, non-protective CD4 T cells during tuberculosis. Cell Rep. 18, 3091-3104 (2017).

10. Sullivan, B.M. et al. Increased susceptibility of mice lacking T-bet to infection with Mycobacterium tuberculosis correlates with increased IL-10 and decreased IFN-gamma production. J. Immunol. 175, 4593-4602 (2005).

11. Torrado, E. et al. Interleukin $27 \mathrm{R}$ regulates $\mathrm{CD} 4+\mathrm{T}$ cell phenotype and impacts protective immunity during Mycobacterium tuberculosis infection. J. Exp. Med. 212, 1449-1463 (2015).

12. Andersen, P. \& Urdahl, K.B. TB vaccines; promoting rapid and durable protection in the lung. Curr. Opin. Immunol. 35, 55-62 (2015).

13. Orr, M.T. et al. Interferon gamma and tumor necrosis factor are not essential parameters of CD4 + T-cell responses for vaccine control of tuberculosis. J. Infect. Dis. 212, 495-504 (2015).

14. Woodworth, J.S., Aagaard, C.S., Hansen, P.R., Cassidy, J.P., Agger, E.M. \& Andersen, P. Protective CD4 T cells targeting cryptic epitopes of Mycobacterium tuberculosis resist infection-driven terminal differentiation. J. Immunol. 192, 3247-3258 (2014).

15. Woodworth, J.S. et al. Subunit vaccine H56/CAF01 induces a population of circulating CD4 T cells that traffic into the Mycobacterium tuberculosisinfected lung. Mucosal Immunol, 10, 555-564 (2017).

16. Russell, D.G., Barry, C.E. 3rd \& Flynn, J.L. Tuberculosis: what we don't know can, and does, hurt us. Science 328, 852-856 (2010).

17. Capuano, S.V. 3rd et al. Experimental Mycobacterium tuberculosis infection of cynomolgus macaques closely resembles the various 
manifestations of human M. tuberculosis infection. Infect. Immun. 71, 5831-5844 (2003).

18. Mattila, J.T. etal. Microenvironments in tuberculous granulomas are delineated by distinct populations of macrophage subsets and expression of nitric oxide synthase and arginase isoforms. J. Immunol. 191, 773-784 (2013).

19. Mehra, S. et al. Granuloma correlates of protection against tuberculosis and mechanisms of immune modulation by Mycobacterium tuberculosis. J. Infect. Dis. 207, 1115-1127 (2013).

20. Lin, P.L. et al. Tumor necrosis factor neutralization results in disseminated disease in acute and latent Mycobacterium tuberculosis infection with normal granuloma structure in a cynomolgus macaque model. Arthritis Rheum. 62, 340-350 (2010).

21. Esmail, $\mathrm{H}$. et al. Characterization of progressive HIV-associated tuberculosis using 2-deoxy-2-[18F]fluoro-D-glucose positron emission and computed tomography. Nat. Med. 22, 1090-1093 (2016).

22. Lin, P.L. et al. Sterilization of granulomas is common in active and latent tuberculosis despite within-host variability in bacterial killing. Nat. Med. 20 , 75-79 (2014).

23. Gideon, H.P. et al. Variability in tuberculosis granuloma T cell responses exists, but a balance of pro- and anti-inflammatory cytokines is associated with sterilization. PLoS Pathog. 11, e1004603 (2015).

24. Arlehamn, C.L. et al. Transcriptional profile of tuberculosis antigen-specific Tcells reveals novel multifunctional features. J. Immunol. 193, 2931-2940 (2014).

25. Lindestam Arlehamn, C.S. et al. Memory T cells in latent Mycobacterium tuberculosis infection are directed against three antigenic islands and largely contained in a CXCR3 + CCR6 + Th1 subset. PLoS Pathog. 9, e1003130 (2013).

26. Mothe, B.R. et al. The TB-specific CD4(+) Tcell immune repertoire in both cynomolgus and rhesus macaques largely overlap with humans. Tuberculosis (Edinb) 95, 722-735 (2015).

27. Lin, P.L. et al. Early events in Mycobacterium tuberculosis infection in cynomolgus macaques. Infect. Immun. 74, 3790-3803 (2006).

28. Reiley, W.W. et al. Distinct functions of antigen-specific CD4 T cells during murine Mycobacterium tuberculosis infection. Proc. Natl Acad. Sci. USA 107, 19408-19413 (2010).
29. Russell, D.G., Cardona, P.J., Kim, M.J., Allain, S. \& Altare, F. Foamy macrophages and the progression of the human tuberculosis granuloma. Nat. Immunol. 10, 943-948 (2009).

30. Davis, J.M. \& Ramakrishnan, L. The role of the granuloma in expansion and dissemination of early tuberculous infection. Cell 136, 37-49 (2009).

31. Gormus, B.J., Blanchard, J.L., Alvarez, X.H. \& Didier, P.J. Evidence for a rhesus monkey model of asymptomatic tuberculosis. J. Med. Primatol. 33 , 134-145 (2004).

32. Anderson, K.G. et al. Intravascular staining for discrimination of vascular and tissue leukocytes. Nat. Protoc. 9, 209-222 (2014).

33. Gerlach, C. et al. The chemokine receptor CX3CR1 defines three antigenexperienced CD8 T cell subsets with distinct roles in immune surveillance and homeostasis. Immunity 45, 1270-1284 (2016).

34. Sallusto, F. Heterogeneity of human CD4 $(+) T$ cells against microbes. Annu. Rev. Immunol. 34, 317-334 (2016).

35. Acosta-Rodriguez, E.V. et al. Surface phenotype and antigenic specificity of human interleukin 17-producing Thelper memory cells. Nat. Immunol. 8, 639-646 (2007).

36. Paroni, M. et al. Recognition of viral and self-antigens by $\mathrm{TH} 1$ and $\mathrm{TH} 1 / \mathrm{TH} 17$ central memory cells in patients with multiple sclerosis reveals distinct roles in immune surveillance and relapses. J. Allergy Clin. Immunol.; doi:org/ 10.1016/j.jaci.2016.11.045 (2017).

37. Ramstein, J. et al. IFN-gamma-producing T-helper 17.1 cells are increased in sarcoidosis and are more prevalent than T-helper type 1 cells. Am. J. Respir. Crit. Care Med. 193, 1281-1291 (2016).

38. Duhen, T. \& Campbell, D.J. IL-1beta promotes the differentiation of polyfunctional human CCR6 + CXCR3 + Th1/17 cells that are specific for pathogenic and commensal microbes. J. Immunol. 193, 120-129 (2014).

39. Okada, S. et al. Immunodeficiencies. Impairment of immunity to Candida and Mycobacterium in humans with bi-allelic RORC mutations. Science 349, 606-613 (2015).

40. Coleman, M.T. et al. PET/CT imaging reveals a therapeutic response to oxazolidinones in macaques and humans with tuberculosis. Sci. Transl. Med. 6, 265ra167 (2014). 